\title{
The Bitvector Machine: A Fast and Robust Machine Learning Algorithm for Non-linear Problems
}

\author{
Stefan Edelkamp and Martin Stommel \\ Research Group Artificial Intelligence, Universität Bremen \\ Am Fallturm 1, 28359 Bremen, Germany \\ \{edelkamp, mstommel\}@tzi.de
}

\begin{abstract}
In this paper we present and evaluate a simple but effective machine learning algorithm that we call Bitvector Machine: Feature vectors are partitioned along component-wise quantiles and converted into bitvectors that are learned. It is shown that the method is efficient in both training and classification. The effectiveness of the method is analysed theoretically for best and worst-case scenarios. Experiments on high-dimensional synthetic and real world data show a huge speed boost compared to Support Vector Machines with RBF kernel. By tabulating kernel functions, computing medians in linear-time, and exploiting modern processor technology for advanced bitvector operations, we achieve a speed-up of 32 for classification and 48 for kernel evaluation compared to the popular LIBSVM. Although the method does not generally outperform a SVM with RBF kernel it achieves a high classification accuracy and has qualitative advantages over the linear classifier.
\end{abstract}

Keywords: classification, support vector machine, time/accuracy tradeoff.

\section{Introduction}

Due to the flexibility of kernel functions, statistical machine learning with Support Vector Machines, SVMs for short [1, is one of the most successful approaches for classification applications [2].

The number of support vectors learned affects training and classification speed. Bordes et al. ([3]) assume that not all training samples are equally relevant for the resulting model. Their LASVM 11 implementation achieves a significant speedup during training by using an online approach, where the margin is determined from a small subset of the input data. Important modeling decisions can be reached even without taking into account the class label of a data point.

The measurement of the model accuracy also affects the training speed. Joachims' 4. SVMLIGHT2 implementation achieves a significant speed-up in

${ }^{1}$ http://leon.bottou.org/projects/lasvm

2 http://svmlight.joachims.org/

P. Flach et al. (Eds.): ECML PKDD 2012, Part I, LNCS 7523, pp. 175-190, 2012.

(C) Springer-Verlag Berlin Heidelberg 2012 
the estimation of the leave-one-out error by evaluating the Lagrange multipliers and slack variables of a trained model. This saves the retraining of the model for every sample left out. Tsang et al. 5] propose a radical simplification of the model by approximating the convex hull of the training data by an enclosing ball in the high-dimensional feature space. The allowed error of the approximation is a parameter of the algorithm. The method works iteratively to find the centre of the ball. The idea of using a reduced set of support and also non-support vectors to define the decision border has also been used in previous approaches [67]. Influences stem for example from approximate k-nearest neighbours [8] or computational geometry [7].

There are also fast SVM implementations with linear kernels that exceed the library used in this paper (LIBSVM 3 ) by far, e.g. LIBLINEAR 4 and Leon Bottou's stochastic gradient descent SVM5. However, for complex data where single classes comprise multiple distant sub-clusters, non-linear kernels achieve a higher accuracy. Chang et al. [9] achieve a speed-up of a second order polynomial classifier by using optimisation techniques usually reserved for linear kernels. Zhang et al. 10 compute a low rank linear approximation of the kernel matrix of a non-linear kernel and evaluate it by a linear SVM.

The dimensionality of the input data affects the speed of the classification as well, but it also often causes a numerical instability known as the curse of dimensionality. It is described [11] as a general unreliability of distance computations for data sets where minimum and maximum distances approximate with rising dimensionality. The effect can be reduced by choosing a smaller norm than the Euclidian [12, but taking high roots is numerically difficult, too.

In the application of machine learning algorithms to image and video data, a binary discretisation of the popular SIFT (Scale Invariant Feature Transformation [13.) feature vector does not suffer from this effect [14, whereas the original SIFT representation does. Although a feature binarisation is a dramatic simplification of the input data, it has been observed for SIFT and SURF (Speeded Up Robust Features [15]) descriptors that the matching accuracy does not decrease significantly [16]. In some cases the relative error rates even decreased. Moreover, the length of the descriptors is reduced by a factor of 8 for SIFT (128 dimensions, usually implemented as single bytes) and by a factor of 32 for SURF (64 dimensions, usually implementated by 4 byte floating point values). These advantages are highly relevant for robotics applications as SURF or SIFT are called at a high frequency in Simultaneous Localisation and Mapping (SLAM)6.

In this paper, we study the findings on feature binarisation in a more general setting. A brief summary of SVMs allows us to introduce the method under the

\footnotetext{
3 http://www.csie.ntu.edu.tw/ cjlin/libsvm

4 http://www.csie.ntu.edu.tw/ cjlin/liblinear

5 http://leon.bottou.org/projects/sgd (this algorithm has been ported by us to the GPU for an even faster evaluation time)

${ }^{6}$ http://openslam.org
} 
notion of a Bitvector Machin 7 . We discuss conditions under which the binarisation of a feature vector is appropriate. We observe that the Hamming distances between binarised input vectors are discrete and limited. This allows a number of important code optimisations for kernel evaluation, most notably the use of look-up tables and native processor instructions. In contrast to previous work [16 where no dedicated CPU instructions are used, we can therefore quantify the main advantage of the method: the speed in the kernel evaluation. For transforming the input data from floating point to binary strings we exploit that medians can be computed efficiently. By replacing the medians by $q$-quantiles, we generalise the Bitvector Machine to a Multi-Bitvector Machine. The method allows for a wider range of possible time-accuracy trade-offs and avoids some worst-case behaviours of the single-bit binarisation. In the empirical part of the paper we evaluate the (single-bit) approach in three different experimental settings. The first and second ones comprise artificial data (a Mixed Gaussian Distribution). The third one deals with real-world data from a face recognition application. As a result we achieve a several fold speed-up in the classification with only a small loss in accuracy compared to LIBSVM. As far as pure kernel computations are concerned, the method also accelerates training, although this is not deepened in the paper.

\section{Support Vector Machines}

Raw data presented to a supervised statistical machine learning algorithm [17] can be arbitrarily complex and is often mapped to a set of numerical values, called the feature vector. The classification problem deals with the prediction of the label $l$ of previously unknown feature vectors $\mathbf{x} \in \mathbb{R}^{d}$ that constitute the test data. During training, a partitioning of the feature space $\mathbb{R}^{d}$ is learned, where each partition is assigned a label $l$ from a small set $L$ based on a set of training samples $\left(\mathbf{x}_{1}, l_{1}\right), \ldots,\left(\mathbf{x}_{k}, l_{k}\right) \in \mathbb{R}^{d} \times L$ with known labels. The challenge is to approximate the unknown distribution without overfitting the training data.

Support Vector Machines. [1], SVMs for short, achieve this task by learning coefficients for a kernel mapping to a high-dimensional space, where a linear class border is spanned up by a number of support vectors that outline the data. We keep the presentation brief as there are text books on SVMs and related kernel methods [2]8]. Theoretically, it should be sufficient to determine the class border by just three support vectors. However, it is not known in advance if any of the known kernels realises a suitable mapping. The use of generic kernels instead leads to a much larger number of support vectors (which critically influence classification time). In the worst case finding a separating hyperplane takes quadratic time in the number of data points.

The classification rule for a two-class non-linear classification function $\phi$ is

$$
f(\mathbf{x})=\operatorname{sign}\left(\sum_{i=1}^{s} \beta_{i}\left(\phi\left(\mathbf{x}_{i}\right) \cdot \phi(\mathbf{x})\right)+b\right),
$$

\footnotetext{
7 The term machine links to the fact that the classifier realises a mathematical function, sometimes referred to as a machine.
} 
where $\mathbf{x}_{i}$ is the $i$ th of $s \leq k$ support vectors, $\beta_{i}$ is a coefficient that includes class label and Lagrange multiplier from the optimisation, and $b$ is some additional translation constant. Assuming a kernel function $K(\mathbf{u}, \mathbf{v})=\phi(\mathbf{u}) \cdot \phi(\mathbf{v})$ we get

$$
f(\mathbf{x})=\operatorname{sign}\left(\sum_{i=1}^{s} \beta_{i} \cdot K\left(\mathbf{x}_{i}, \mathbf{x}\right)+b\right),
$$

which does not refer directly to $\phi$, known as the kernel trick.

SVM training is a convex optimisation problem which scales with the training set size rather than the feature space dimension. While this is usually considered to be a desired quality, in large scale problems it may cause training to be impractical and classification to be time consuming.

The corpus of SVM applications is large and encompasses many areas of computer science [2]. As kernel functions can be complex, the application of SVMs is large and kernels can be designed to cover simple regression to neural network approaches [19] and time series [20. However, linear kernels $K(\mathbf{u}, \mathbf{v})=\mathbf{u} \cdot \mathbf{v}+g$ or Gaussian (RBF) kernels $K(\mathbf{u}, \mathbf{v})=\exp \left(-\gamma\|\mathbf{u}-\mathbf{v}\|^{2}\right), \gamma \in \mathbb{R}$ are the ones that are most commonly used. In the second case, a SVM is a function that itself defines a Mixed Gaussian Distribution [21] and is related to Radial Basis Networks and Neuronal Nets with one hidden layer. Since the optimisation objective is high accuracy instead of a low number of support vectors, a Mixed Gaussian input will be usually modeled by multiple centres per Gaussian in the SVM.

The running time for classifying one vector is $O(s d e)$, where $e$ is the time to evaluate the exponential. Practical SVM implementations might assume the input data to be normalised to avoid numerical difficulties.

\section{Bitvector Machine}

A Bitvector Machine, BVM for short, is an SVM with a binarisation (Boolean discretisation) of the input: All vectors $\mathbf{x}_{i} \in \mathbb{R}^{d}, 1 \leq i \leq k$, used in the training phase and all vectors evaluated in the test phase are mapped to $\{0,1\}^{d}$. The labels remain unchanged. The results are then fed into a SVM.

The median of $k$ totally ordered elements is the element in the $\lfloor k / 2\rfloor$-th position after sorting. Median selection can be performed in linear time [22]. Let $\overline{\mathbf{x}}=\left(\bar{x}^{1}, \bar{x}^{2}, \ldots, \bar{x}^{d}\right)^{\top} \in \mathbb{R}^{d}$ be the component-wise median of the input vector, i.e. $\bar{x}^{j}$ is the median of the $j$-th vector components of $\mathbf{x}_{1}, \ldots, \mathbf{x}_{k}$.

The BVM maps (training and test) vectors $\mathbf{x} \in \mathbb{R}^{d}$ to binary strings $\mathbf{z}=$ $\left(z^{1}, z^{2}, \ldots, z^{d}\right)^{\top} \in\{0,1\}^{d}$ as follows: For each $j, 1 \leq j \leq d$, we have $z^{j}=0$ if and only if $x^{j}<\bar{x}^{j}$. Otherwise $z^{j}$ is set to 1 .

Theorem 1 (Time Complexity Single-Bit Binarisation). The binarisation of all $\mathbf{x}_{i} \in \mathbb{R}^{d}, 1 \leq i \leq k$, takes time $\Theta(k d)$.

Proof. Computing all component-wise medians of vectors $\mathbf{x}_{i} \in \mathbb{R}^{d}, 1 \leq i \leq k$, i.e. $\overline{\mathbf{x}}$, requires $O(k d)$ time. Thresholding all vectors $\mathbf{x}_{i} \in \mathbb{R}^{d}$ component-wise with $\overline{\mathbf{x}}$ can be executed in time $O(k d)$. Considering the input size of the data, the time $O(k d)$ is optimal. 
By using $q$ quantiles instead of the medians (where $q=2$ ) we can create more detailed feature representations of longer word length. Quantiles represent a subdivision of the domain of a random variable into $q$ consecutive partitions of equal cumulative density $1 / q$. The respective thresholds can be read from the cumulative distribution function or by recursively computing medians (if $q$ is a power of 2). The assignment of a random value to its $\eta$-th, $1 \leq \eta \leq q$, quantile can be done in log-time by arranging the thresholds in a balanced binary tree. The representation of the index $\eta$ of a quantile requires $m=\lceil\lg q\rceil$ bits.

A Multi-Bitvector Machine, MBVM for short, maps (training and test) vectors $\mathbf{x} \in \mathbb{R}^{d}$ to binary strings $\mathbf{z}=\left(z^{1}, z^{2}, \ldots, z^{m d}\right)^{\top} \in\{0,1\}^{m d}, m=\lceil\lg q\rceil$. In order to binarise a $d$-dimensional feature vector, we compute quantiles of fixed $q$ independently for every dimension. The binary feature vector is the concatenation of all $d$ quantile indices $\eta$, the resulting length is thus $m d$ bits. Computing all component-wise quantiles of vectors $\mathbf{x}_{i} \in \mathbb{R}^{d}, 1 \leq i \leq k$, requires $O(m k d)$ time, as computing the list of all quantiles of a $k$ element set takes time $O(m k)$ (see Ex. 9.3-6, p. 223 in [23]). As $q<k$ and, subsequently, $m<\lceil\lg k\rceil$ this approach is still faster than sorting with its time complexity of $\Omega(k \lg k)$.

Corollary 1 (Time Complexity Multi-Bit Binarisation). The multi-bit binarisation of all $\mathbf{x}_{i} \in \mathbb{R}^{d}, 1 \leq i \leq k$, using q-quantiles takes time $O(m k d)$, with $m=\lceil\lg q\rceil$.

Instead of reducing the dimension as done in related work, e.g. on random projections 24, we enlarge it. The motivation is that the increase in dimensionality is compensated by the speed-up by the lower bit-rate.

The computation of median (quantile) based binary representation corresponds to a partitioning and re-labeling of the feature space. If we were to split the data iteratively, we would build a kd-tree in $O(n \lg n)$ time [25, for which rectangular range queries take $O(\sqrt{n}+k)$ and membership queries take $O(\lg n)$ time [26]. In contrast, in the BVM the median splits are chosen independently of each other, one in each vector component. The binarisation therefore defines a partitioning of the feature space into regions, where all separating hyperplanes intersect in one point. Geometrically, it can be interpreted as moving the origin of the Cartesian coordinate system to $\overline{\mathbf{x}}$ and representing each resulting orthant by a bitvector $\{0,1\}^{d}$ that indicates the position relative to the iso-oriented hyperplanes. The bitvectors correspond to nodes in a $d$ dimensional hypercube (an edge in the hypercube has Hamming Distance 1).

Let $\psi$ be the mapping that performs the binarisation. We have

$$
f(\mathbf{x})=\operatorname{sign}\left(\sum_{i=1}^{t} \delta_{i} \cdot K\left(\psi\left(\mathbf{x}_{i}\right), \psi(\mathbf{x})\right)+g\right) .
$$

Due to a different training, the number of support vectors $t$, weights $\delta$ and bias $g$ might be different from the original values $(s, \beta, b$ in Eq.2). Moreover, as $K(\psi(\mathbf{u}), \psi(\mathbf{v}))=\phi(\psi(\mathbf{u})) \cdot \phi(\psi(\mathbf{v}))$, we see that we are actually dealing with a different kernel that transforms data via $\phi \circ \psi$ from $\mathbb{R}^{d}$ first into the Boolean space $\{0,1\}^{d}$ before lifting it into higher dimensions. 
Because of the symmetry property, distance metrics based on an element by element comparison (like Euclidean or Hamming distance) in $\{0,1\}^{d}$ yield only $d+1$ different values. All possible results of the kernel $K\left(\psi\left(\mathbf{x}_{i}\right), \psi(\mathbf{x})\right)$ can therefore be precomputed.

For the Gaussian kernel $K(\psi(\mathbf{u}), \psi(\mathbf{v}))=\exp \left(-\gamma\|\psi(\mathbf{u})-\psi(\mathbf{v})\|^{2}\right)$ the term $\|\psi(\mathbf{u})-\psi(\mathbf{v})\|^{2}$ can only yield $d+1$ different values. For the Euclidean norm they range from 0 to $d$ and equal the Hamming distance of the kernel arguments. By applying the parameter $\gamma$ and the exponential to these values, the whole kernel can be precomputed and stored in a table. This avoids the repeated time consuming computation of the exponential during classification. The Hamming distance can be computed by applying the population count instruction (counting the number of bits set) to the bitwise XOR disjunction of the arguments.

Precomputing kernels reduces training and classification time. Because training is done only once, we focus on the latter.

Theorem 2 (Time Complexity Single-Bit Classification). Assuming d to be $O(w)$ for the computer word width $w$ and native population count, the running time for classifying one bitvector is $O(t+d)$, where $t$ is the number of support vectors.

If population count is not native on the word level, then the classification of one vector has the complexity $O\left(d+t \lg ^{*} d\right)$, where $\lg ^{*} d$ is the iterated logarithm, i.e. the height of the shortest tower of powers $2^{2 \cdots}$ that equals or exceeds $d$.

Proof. Computing the binarisation $\psi(\mathbf{x})$ of the test vector $\mathbf{x}$ takes time $O(d)$. The population count and XOR to be executed on the word level to compute the Hamming Distance run in $O(1)$. Given that the kernel is tabulated, we require only lookups to the kernel table, so that multiplication with a constant and addition have to be executed $t$ times to evaluate the classification formula $\sum_{i=1}^{t} \delta_{i} \cdot K\left(\psi\left(\mathbf{x}_{i}\right), \psi(\mathbf{x})\right)$.

For larger values of $d$ population count can be done in $O\left(\lg ^{*} d\right)$ by iterating the HAKMEM algorithm 8 .

The second part of the theorem assumes large word width $w$ and is mainly of theoretical interest.

Assuming that the binary representation of the bitvectors due to computing the quantiles still fits into a computer word width $w$, for an MBVM computing the binarisation of the test vector $\mathbf{x}$ takes time $O(m d)$. Hence, the running time for classification generalizes to $O(t+m d)$ with $m$ being the dual logarithm of the number of quantiles considered.

Corollary 2 (Time Complexity Multi-Bit Classification). Assuming d to be $O(w)$ for the computer word width $w$ and native population count, the running time for classifying one bitvector is $O(t+m d)$, where $t$ is the number of support vectors.

If population count is not native on the word level, then the classification of one vector has the complexity $O\left(m d+t \lg ^{*}(m d)\right)$.

${ }^{8}$ David Eppstein, http://11011110.livejournal.com/38861.html 
The entropy for one split along the median is certainly maximal as long as class labels are not taken into account. But even for simplified Mixed Gaussian Distributions (2D, shifted mean, same deviation but same amplitude) entropies can only be approximated [27.

The number of regions distinguishable by the BVM rises exponentially with dimensionality. For dimensionality $d$ we have $2^{d}$ possible regions. As we split the data component-wise, the BVM corresponds to a static decision tree that has depth $d$ and that is independent of the number of elements. An explicit construction of such a tree, however, is not required. In contrast to SVMs, the binarisation automatically normalises the input data to the unit hypercube.

Because the BVM is trained the same way as a SVM, we can still use crossvalidation or leave-one-out-validation to estimate the classification error.

\section{Case Studies}

One question is if and when the binary kernel is better than a linear one. If we assign each vector $\{0,1\}^{n}$ with even population count with class 1 and each vector $\{0,1\}^{n}$ with odd population count with class 2 , then we generalise the XOR problem to higher dimension (the minimal Hamming distance of two elements class in one class is two). This is clearly not linearly separable, but the BVM can find a perfect classification.

This clearly is a best-case scenario, but we can argue that linearly nonseparable but binary separable examples are common in practice. One reason for this is that many classes underly the principles of differentiation and composition. Let us assume for example a set of images of noses, either taken from a left angle or from a frontal perspective. Although left noses are visually and numerically similar to each other, they differ strongly from frontal noses and occupy a separate region in feature space. The combined class nose, however, is activated by features from both differentiations. The dispersed placement of multiple clusters of sub-classes in feature space can easily create non-linearly separable situations, especially in multi-class problems. Moreover, real-world data often comes from independent or principle component analyses. As a result, feature distributions for different sub-classes tend to be aligned with the coordinate axes.

One worst-case scenario for the BVM in two dimensions is a checker-board layout of two classes because after two orthogonal cuts all fields of the checkerboard that fall into the same quadrant are represented by the same bitvector and with it the same class. For this theoretical setting, the SVM calls for several support vectors and likely an overfitting of the data. If the number of Gaussian kernels is small and the dimension is high, the BVM has good chances to find a discriminative partitioning. As a result, feature binarisation preserves high selectivity and lifts the curse of dimensionality.

However, if we were to use a MBVM with as many quantiles as the checkerboard size, the worst-case behavior would be avoided and we would encounter a best-case scenario with accurate class boundaries. 
Even though entire orthants collapse to single data points on the hypercube, the Gaussian weighting of the binary vectors still preserves at least some geometrical meaning. The weights for a specific class are propagated nonlinearly from one orthant to another via shared hyperedges. For shared hyperedges the Hamming distance of the associated bitvectors is one.

\section{Experiments}

We performed the experiments on one core of a desktop computer (model Intel Core i7 920 CPU 2.67 GHz) running Ubuntu 10.10 (Linux kernel 2.6.32-23generic) with 24 GB main memory. With such memory capacity, there was no need to use virtual memory. We compiled all programs using GNU C++ compiler (gcc version 4.3 with option -03 and -mpopcnt).

For the implementation of the BVM we have extended LIBSVM to support bitvector manipulation based on a precomputed kernel and native population counting. LIBSVM is chosen because it is well known, widely used, and easily extendible. It uses a one-versus-one strategy for multi-class problems.

The use of SVM implementations aiming at higher training speed [34] would not provide a deeper insight because our method aims at testing speed. The BVM is also not in contradiction to approximative methods working on reduced sets 56678 . It would therefore be possible to combine a fast binary vector representation with a small approximated set of support vectors in order to achieve an even higher speed-up. However, in favour of the clarity of the presentation we refrain from such combined approaches.

We evaluate the BVM in two synthetic and one natural scenarios.

\subsection{Artificial Data}

To validate our ideas experimentally, we produced training and test data for two and five-class problems in a random process with defined statistical properties. For each class we realised a sampling of a Mixture of Gaussians. The mean $\mu_{i}, i=1,2, \ldots$ of each multivariate Gaussian

$$
p_{i}(\mathbf{x})=\frac{1}{(2 \pi)^{\frac{d}{2}}|\Sigma|^{\frac{1}{2}}} \exp \left(-\frac{1}{2}\left(\mathbf{x}-\mu_{i}\right)^{\top} \Sigma^{-1}\left(\mathbf{x}-\mu_{i}\right)\right)
$$

is placed in the unit hypercube. The bandwidth is set globally in the main diagonal of the covariance matrix $\Sigma$ for all Gaussians. The number of Gaussians is set individually for every experiment.

The maximum likelihood estimate (Bayes) of the Mixed Gaussian distribution is used as the ground truth to which an SVM and the proposed method are compared. Linear kernels are used to detect linearly separable situations.

The difficulty of the created problems is controlled by adjusting the bandwidth of the Gaussians for a specific accuracy of the maximum likelihood estimator. If we keep the number of Gaussians fixed and increase the dimensionality, we have to increase the bandwidth, too. Otherwise the overlap between the 
Gaussians decreases and the difficulty of the problem changes. The bandwidth of the kernel is therefore a parameter of the experimental setting. It exhibits a strong influence on the resulting estimate. We therefore set it manually, although Silverman indicates a dependency of the bandwidth on the root of the dimensionality 28 .

First Scenario. As a parameter of the synthetic experiment, we choose the bandwidth to provide accuracy values to be in the range $[0.8,0.85]$. For determining the classification accuracy (on the training and test sets) we conducted two experiments, one for a two-class problem and one for a five-class problem both with rising dimension (dimensionalities $2,4,8, \ldots, 256$ ). The two-class problem is modeled by three Gaussians per class at uniformly distributed random positions in the unit cube. With 5 Gaussians per class, the five-class problem is more complex. Each Gaussian is sampled 70 times. The data set is randomised and split into equally sized training and test sets. The parameters $C$ (a weighting of the slack variables in the optimised function) and $\gamma$ of both the BVM and the RBF kernel of LIBSVM are optimised in a grid search using the Python scripts provided by LIBSVM. For the Gaussian kernel and the BVM the training set is further subdivided in order to perform a five-fold cross validation for parameter selection.

The plots in Fig. 1 show that the BVM solves the problem surprisingly well, given the limitation that the BVM does not represent any gradual or continuous feature values.

For smaller dimensions and the two-class problem, all methods lead to very similar results with a small advantage for the SVM with Gaussian kernel. The results of the BVM are comparable to those of the linear classifier, with changing winners. For the five-class problem, the results are clearer. Training and test results are closer for smaller dimensionalities and the BVM achieves results between the SVM with Gaussian and linear kernel, although closer to the linear one. The recognition rate of the BVM has a maximum at 32 dimensions. It seems that lower dimensions limit the capacity to represent information.

For more than 32 dimensions, the accuracy drops significantly for all methods. The pronounced divergence between the training and test results of the linear classifier indicates heavy overfitting. The Gaussian kernel does not show this overfitting but the bad results indicate a clear failure, too. Although the crossvalidation on subsamples of the training set leads to better estimates of the final recognition rate, it cannot compensate for missing information.

In summary the accuracy of all classifiers is limited, so our experiment may be overly complex. Even the linear kernel might be competitive if additional application constraints are taken into account. It must also be said that the difficult class borders in this Gaussian mixture do not have much in common with data sets from pattern recognition tasks: In such applications, the feature vector often represents the similarity of a query object to a set of prototypes, where each similarity is stored in one dimension. Consequently, the class border would not normally cross a coordinate axis multiple times. The effect is even reinforced by the common use of coordinate transforms such as principle component analysis. 

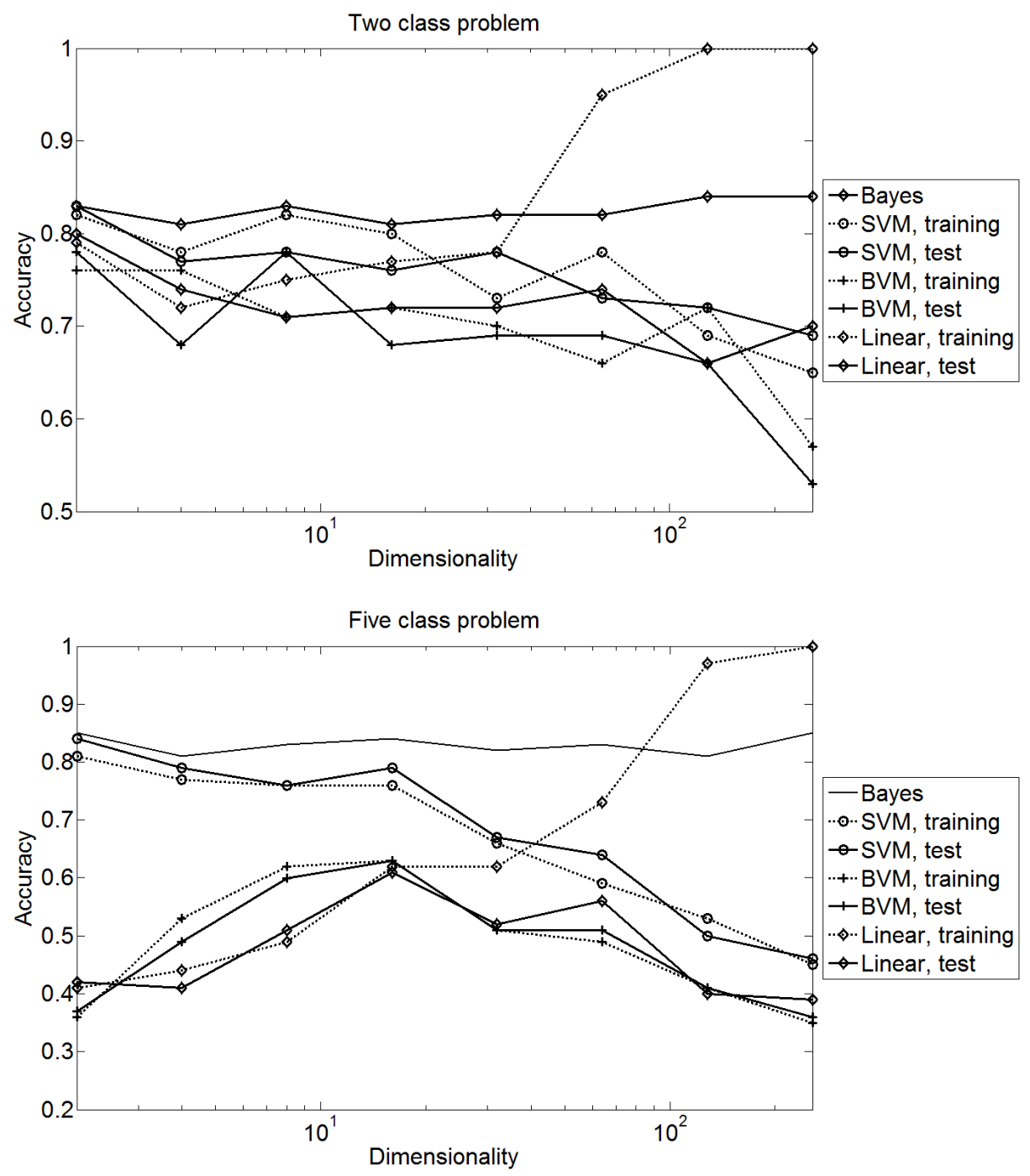

Fig. 1. First scenario: Accuracy for the SVM (RBF and linear kernel) and the BVM

In matters of CPU time we see a drastic decrease in computation time for the BVM. Figure 2 shows that we obtain a huge speed-up that additionally increases with the dimension of the problem. The maximum increase of performance was a factor of 32 for the two class problem in 128 dimensions. The increase in CPU time at $d=256$ for the BVM can be explained by the increased word length. Here all word level operations have to be executed four times on a 64 bit machine. For bigger dimensions, computer architecture and compiler must be taken stronger into account. We have not measured the time for the preprocessing step. 

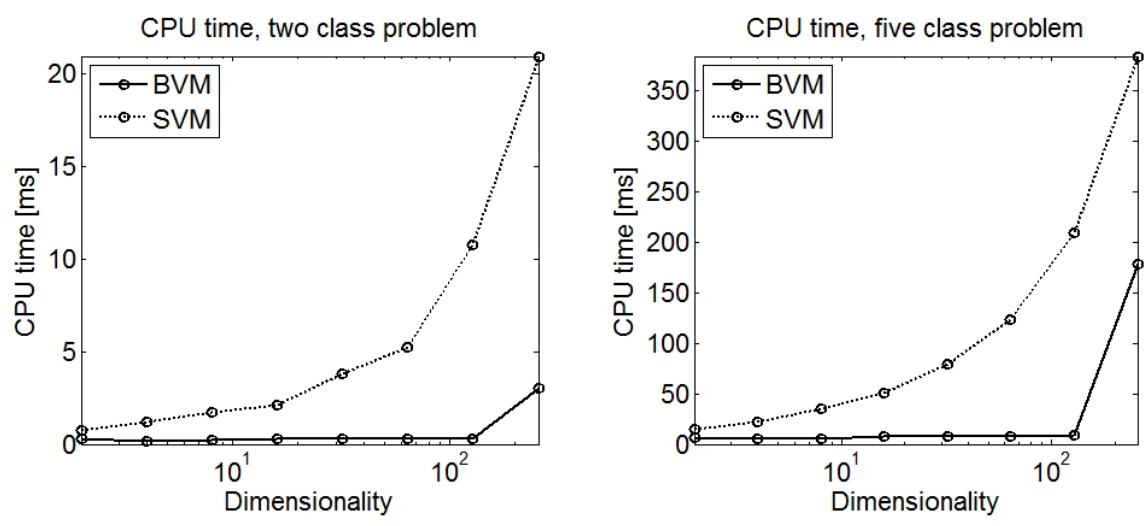

Fig. 2. CPU time measured for the classification of the data sets in the first scenario. The original real valued data is classified by an SVM with Gaussian kernel. The binary data refers to the BVM.

However, it seems obvious to us that a loop over $d$ dimensions in the binarisation of a feature descriptor is uncomparably faster than the computation of dot products to thousands of $d$-dimensional support vectors in the classification of one descriptor.

The Second Scenario represents the above-mentioned high-dimensional XOR problem but with noisy data. To this end, we centre the Gaussians of our Gaussian Mixture at the corners of the unit hypercube and assign class labels to the Gaussians as described above. The bandwidth is adjusted so that the Bayes classifier achieves an accuracy of $90 \%-92 \%$ using the known distribution.

Figure 3 shows the results of the proposed method and the SVM using the RBF and linear kernel. The Gaussian Mixture consists of four planar XOR problems placed at random sides of the unit hypercube of dimensionality $8,16, \ldots$, 256). A planar XOR problem consists of four Gaussians at the corners of a square with diagonally different class labels. Each Gaussian is sampled 70 times. The best results can be seen for the SVM using the RBF kernel. The output of the BVM follows the SVM at a lower level. The linear classifier fails completely because it cannot represent the non-linear class borders.

Figure 4 shows the results for an 8-dimensional XOR arrangement of varying sparseness. For this distribution, Gaussians are randomly placed in a certain percentage of all corners and sampled 100 times each. For a sparse filling of $10 \%$, the distribution still seems linearly separable. However, with increasing density the linear classifier quickly approaches random, whereas the BVM improves gradually and finally outperforms the SVM with RBF kernel (corner fill factor $\geq 0.5)$.

This good result shows that the interpretation of the BVM as a simplified approximation of the SVM depends on the understanding of the data (and studying the data is always a good start). If it is not known how the data corresponds 


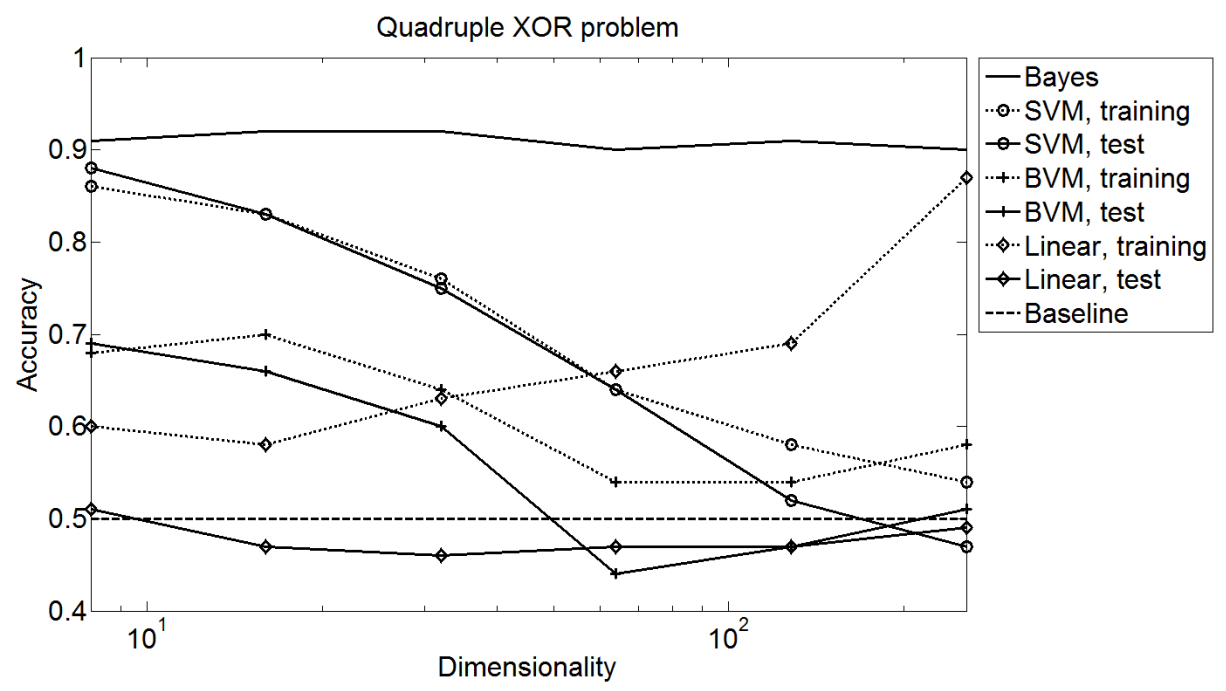

Fig. 3. Second scenario: Accuracy for a two class problem including four planar xor problems

to the described case scenarious, then the BVM must be considered as a nonparametric, simplified method and the results can be inferior to the SVM. If it is known that the data corresponds to a good case scenario, then we use the BVM as a model based method that benefits from our prior knowledge about the data.

\subsection{Real World Data}

The Third Scenario is a Computer Vision task, where SIFT descriptors [13] are classified into 16 classes (15 classes representing different parts of a face plus one background class). Additionally to the original SIFT method, we also tested a variation that takes into account that faces are shown in an upright orientation in most images. Without going into detail, we can say that the original SIFT method is rotationally invariant, i.e. different rotated versions of the image result in approximately the same feature vector. Our variation in contrast (marked as 'absolutely oriented' in Tab.1) is selective to orientation, so different rotated versions of the same visual pattern can be distinguished. In both cases the binarised feature vectors have 128 bits, so they can be compactly stored in two 64-bit words.

We evaluate the effectiveness of population counting on the machine and study the speed-ups obtained for sole kernel evaluations (Table2) and whole vector classifications (Table 3). We compare the LIBSVM implementation with the BVM in two settings, one with a precomputed 16-bit population count lookup-table $\left(2^{16}\right.$ entries), one with the native population count (__builtin_popcountll). We run three examples for each setting to show that the variance in the running times is 


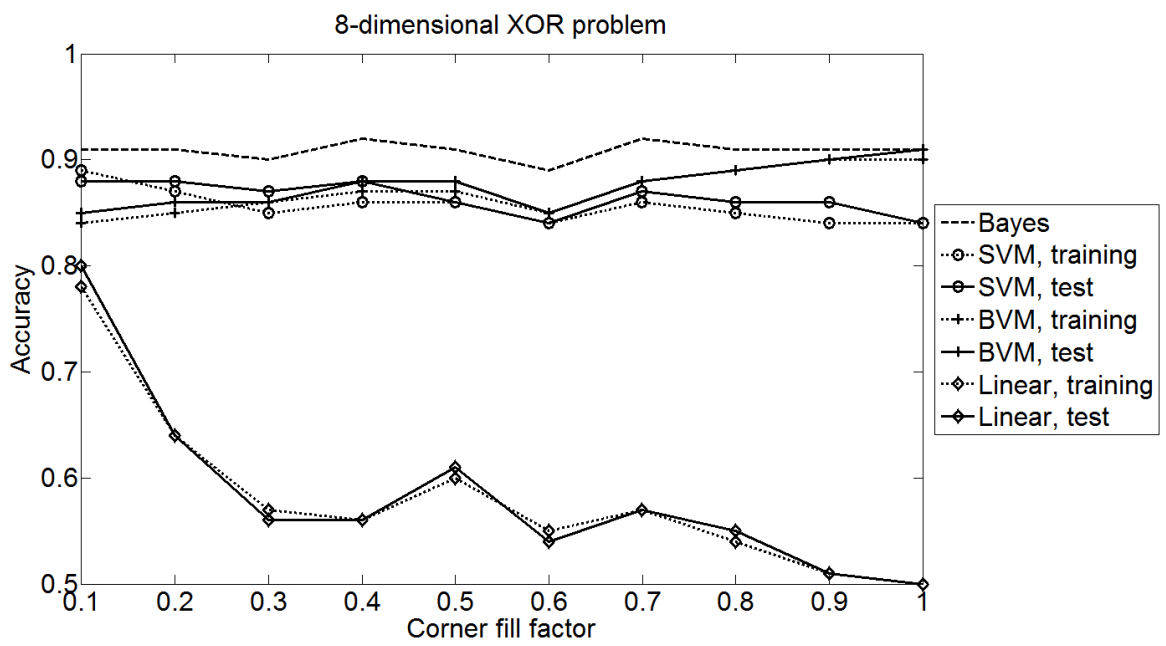

Fig. 4. Accuracy for a non-linear, 8-dimensional class arrangement of increasing complexity

small. The table documents a speed-up factor of 48 in the 116 million kernel comparisons and an improvement by a factor of 17 for the entire classification process. Further speed-ups might be achieved by using a one-versus-all strategy for multiclass problems instead of the one-versus-one strategy implemented in LIBSVM.

The difference in CPU time between the sole kernel computation and the whole classification indicates a strong influence of the code analysis and generation of the compiler, since the number of kernel computations has been equal in both experiments, and the optimisation flags too.

Table 1 shows for different SIFT variations and different class distributions that despite the high speed-up the accuracy of the BVM is on average (from the cross validation) close to that of the SVM. The remaining gap seems statistically significant when comparing the results of the training and test sets. However, it becomes lower with increasing sample size. In comparison to the linear classifier, the BVM is clearly better. For the larger data sets, the BVM is closer to the

Table 1. Accuracy [\%] for SIFT data sets of different size and class distribution. There are 15 foreground classes and one background class. The samples are randomised and split into equally sized training and test sets.

\begin{tabular}{|c|c|c|c|c|c|c|}
\hline Feature vector & SIFT & \multicolumn{5}{|c|}{ SIFT, absolutely oriented } \\
\hline Foreground samples per class & 20 & 20 & 125 & & 250 & \\
\hline Background samples & 2000 & 2000 & 1125 & & 2250 & \\
\hline Classifier & Training Test & Training Test & Training & Test & Training & Test \\
\hline LibSVM, RBF & $\begin{array}{ll}73.4 & 74.0\end{array}$ & $\begin{array}{ll}79.9 & 79.1\end{array}$ & 84.7 & 85.6 & 86.8 & 87.9 \\
\hline BVM, RBF & 67.2 & 75.0 & 82.4 & 83.2 & 84.2 & 85.1 \\
\hline LibSVM, linear & 99.1 & 70.5 & 94.6 & 77.4 & 90.9 & 79.6 \\
\hline
\end{tabular}


Table 2. Evaluation time [s] for 116072232 kernel computations. The first column gives the result for LIBSVM using the Gaussian kernel. The second column gives the results for the BVM, where a look-up-table for 16-bit wide sub-words of the feature vector is used for the computation of the population count. In the last column, the native 64-bit population count CPU instruction is used.

\begin{tabular}{r|c|c|c} 
No & LIBSVM & BVM, 16-bit-popcnt & BVM, 64-bit popcnt \\
\hline 1 & 48.36 & 2.16 & 1.00 \\
2 & 48.27 & 2.16 & 1.00 \\
3 & 48.59 & 2.14 & 1.01
\end{tabular}

Table 3. Time [s] for classifying 16788 vectors in 16 classes, 6914 support vectors

\begin{tabular}{r|c|c|c} 
& \multicolumn{3}{|c}{ Kernel computation using all support vectors } \\
No & LIBSVM & BVM, 16-bit popcnt & BVM, 64-bit popcnt \\
\hline 1 & 39.44 & 4.01 & 2.84 \\
2 & 39.45 & 4.01 & 2.81 \\
3 & 39.71 & 4.00 & 2.71 \\
\hline & \multicolumn{3}{|c}{ only support vectors with $\beta \neq 0$} \\
\hline 1 & & 3.46 & 2.27 \\
2 & & 3.45 & 2.28 \\
3 & & 3.46 & 2.28
\end{tabular}

Gaussian SVM than the linear one. The good results match findings in [16] where the classification accuracy (measured in error rates) has been shown to behave well for other Computer Vision Tasks, too.

\section{Conclusion}

We proposed a machine learning algorithm whose advantages result from a dramatic simplification of the input data. Discretisation certainly has limits in the accuracy of class distributions that are not iso-oriented. We argue, however, that iso-oriented classes with non-linearly separable sub-classes occur in many pattern recognition tasks.

The rising number of positive results in discretising feature vectors into bitvectors prior to the learning process shows that the effectiveness and efficiency of the binarisation in the BVM is an exciting phenomenon. Especially for a growing number of dimensions, where we lack a visual interpretation and where unexpected results like the curse of dimensionality have been measured, research might have concentrated on aspects that do not discriminate well. Even though binarisation reduces the information in the input considerably our experiments show that the results are often of acceptable quality, sometimes even better than the original unabstracted input.

Our experiments on synthetic and real data show that the accuracy of the BVM approximates (and in special cases exceeds) the accuracy of a SVM with $\mathrm{RBF}$ kernel better than a linear classifier, but is up to 48 times faster in the 
kernel computation and up to 32 times faster in classification. These results confirm our theoretical proof of the efficiency of classification and binarisation. Compared to a linear classifier, the accuracy of the BVM is usually higher or equal. Furthermore, the BVM has the ability to model non-linearly separable problems where the linear classifier fails. Together with an improved empirical basis we provided insights that increase the understanding of when and why the approach works well, especially for large feature vectors. The BVM therefore allows for a welcome new trade-off between accuracy and running time for nonlinear problems. The use of $q$-quantiles with $q>2$ in the MBVM can improve the accuracy by the cost of time performance. Hence, the approach is expected to be applicable to problems that are not iso-oriented.

\section{References}

1. Vapnik, V.N., Chervonenkis, A.Y.: Theory of Pattern Recognition. Nauka, USSR (1974) (in Russian)

2. Cristianini, N., Shawe-Taylor, J.: An Introduction to Support Vector Machines and Other Kernel-based Learning Methods. Cambridge University Press (2000)

3. Bordes, A., Ertekin, S., Weston, J., Bottou, L.: Fast Kernel Classifiers with Online and Active Learning. Journal of Machine Learning Research 6, 1579-1619 (2005)

4. Joachims, T.: Learning to Classify Text using Support Vector Machines. Kluwer (2002)

5. Tsang, I., Kocsor, A., Kwok, J.T.: Simpler core vector machines with enclosing balls. In: Ghahramani, Z. (ed.) 24th International Conference on Machine Learning (ICML), pp. 911-918. ACM, New York (2007)

6. Burges, C.J.C.: Simplified Support Vector Decision Rules. In: Proceedings of the Thirteenth International Conference on Machine Learning (ICML), pp. 71-77. Morgan Kaufmann (1996)

7. DeCoste, D.: Anytime Interval-Valued Outputs for Kernel Machines: Fast Support Vector Machine Classification via Distance Geometry. In: Proceedings of the International Conference on Machine Learning (ICML), pp. 99-106 (2002)

8. Decoste, D., Mazzoni, D.: Fast query-optimized kernel machine classification via incremental approximate nearest support vectors. In: International Conference on Machine Learning (ICML), pp. 115-122 (2003)

9. Chang, Y.W., Hsieh, C.J., Chang, K.W., Ringgaard, M., Lin, C.J.: Training and Testing Low-degree Polynomial Data Mappings via Linear SVM. Journal of Machine Learning Research 11, 1471-1490 (2010)

10. Zhang, K., Lan, L., Wang, Z., Moerchen, F.: Scaling up Kernel SVM on Limited Resources: A Low-rank Linearization Approach. In: International Conference on Artificial Intelligence and Statistics, AISTATS (2012)

11. Beyer, K., Goldstein, J., Ramakrishnan, R., Shaft, U.: When Is Nearest Neighbor Meaningful? In: Beeri, C., Bruneman, P. (eds.) ICDT 1999. LNCS, vol. 1540, pp. 217-235. Springer, Heidelberg (1998)

12. Aggarwal, C.C., Hinneburg, A., Keim, D.A.: On the Surprising Behavior of Distance Metrics in High Dimensional Space. In: Van den Bussche, J., Vianu, V. (eds.) ICDT 2001. LNCS, vol. 1973, pp. 420-434. Springer, Heidelberg (2000)

13. Lowe, D.G.: Object Recognition from Local Scale-Invariant Features. In: International Converence on Computer Vision (ICCV), pp. 1150-1157 (1999) 
14. Stommel, M., Herzog, O.: Binarising SIFT-Descriptors to Reduce the Curse of Dimensionality in Histogram-Based Object Recognition. In: Ślęzak, D., Pal, S.K., Kang, B.-H., Gu, J., Kuroda, H., Kim, T.-h. (eds.) SIP 2009. CCIS, vol. 61, pp. 320-327. Springer, Heidelberg (2009)

15. Bay, H., Ess, A., Tuytelaars, T., Gool, L.J.V.: Speeded-Up Robust Features (SURF). Computer Vision and Image Understanding 110(3), 346-359 (2008)

16. Stommel, M., Langer, M., Herzog, O., Kuhnert, K.D.: A Fast, Robust and Low BitRate Representation for SIFT and SURF Features. In: Proc. IEEE International Symposium on Safety, Security, and Rescue Robotics, pp. 278-283 (2011)

17. Summa, M.G., Bottou, L., Goldfarb, B., Murtagh, F., Pardoux, C., Touati, M. (eds.): Statistical Learning and Data Science. CRC Computer Science \& Data Analysis. Chapman \& Hall (2011)

18. Schoelkopf, S.: Learning with Kernels. MIT Press (2001)

19. Schneegaß, D., Schäfer, A.M., Martinetz, T.: The Intrinsic Recurrent Support Vector Machine. In: European Symposium on Artificial Neural Networks (ESANN), pp. 325-330 (2007)

20. Gudmundsson, S., Runarsson, T.P., Sigurdsson, S.: Support vector machines and dynamic time warping for time series. In: International Joint Conference on Neural Networks (IJCNN), pp. 2772-2776 (2008)

21. Permuter, H., Francos, J., Jermyn, I.H.: A study of Gaussian mixture models of colour and texture features for image classification and segmentation. Pattern Recognition 39(4), 695-706 (2006)

22. Blum, M., Floyd, R., Pratt, V., Rivest, R., Tarjan, R.: Time bounds for selection. J. Comput. System Sci. 7, 448-461 (1973)

23. Cormen, T.H., Leiserson, C.E., Rivest, R.L., Stein, C.: Introduction to Algorithms, 3rd edn. MIT Press (2009)

24. Voloshynovskiy, S., Koval, O., Beekhof, F., Pun, T.: Random projections based item authentication. In: Proceedings of SPIE Photonics West, Electronic Imaging / Media Forensics and Security, vol. 7254 (2009)

25. Bentley, J.L.: Multidimensional Binary Search Trees Used for Associative Searching. Commun. ACM 18(9), 509-517 (1975)

26. de Berg, M., Cheong, O., van Kreveld, M., Overmars, M.: Computational Geometry Algorithms and Applications, 3rd edn. Springer, Heidelberg (2008)

27. Michalowicz, J.V., Nichols, J.M., Bucholtz, F.: Calculation of Differential Entropy for a Mixed Gaussian Distribution. Entropy 10(5), 200-206 (2008)

28. Silverman, B.W.: Density Estimation for Statistics and Data Analysis. Chapman and Hall, London (1986) 